Article

\title{
Interpolative Reich-Rus-Ćirić and Hardy-Rogers Contraction on Quasi-Partial b-Metric Space and Related Fixed Point Results
}

\author{
Vishnu Narayan Mishra ${ }^{1,+}+\mathbb{D}$, Luis Manuel Sánchez Ruiz ${ }^{2, *,+} \mathbb{D}$ and \\ Pragati Gautam ${ }^{3,+}$ and Swapnil Verma ${ }^{3,+}(\mathbb{D}$ \\ 1 Department of Mathematics, Indira Gandhi National Tribal University, Lalpur, Amarkantak, Anuppur, \\ Madhya Pradesh 484887, India; vishnunarayanmishra@gmail.com \\ 2 ETSID-Departamento de Matematica Aplicada \& CITG, Universitat Politecnica de Valencia, \\ E-46022 Valencia, Spain \\ 3 Department of Mathematics, Kamala Nehru College (University of Delhi), August Kranti Marg, \\ New Delhi 110049, India; pragati.knc@gmail.com (P.G.); swapnilverma1993@gmail.com (S.V.) \\ * Correspondence: Imsr@mat.upv.es \\ + These authors contributed equally to this work.
}

Received: 30 July 2020; Accepted: 13 September 2020; Published: 17 September 2020

\begin{abstract}
The aim of this paper was to obtain common fixed point results by using an interpolative contraction condition given by Karapinar in the setting of complete metric space. Here in this paper, we have redefined the Reich-Rus-Ćirić type contraction and Hardy-Rogers type contraction in the framework of quasi-partial b-metric space and proved the corresponding common fixed point theorem by adopting the notion of interpolation. The results are further validated with the application based on them.
\end{abstract}

Keywords: quasi-partial b-metric space; common fixed point; interpolation; Reich-Rus-Ćirić contraction; Hardy-Rogers contraction

MSC: 46T99; 47H10; 54H25

\section{Introduction}

In the year 1922, Banach [1] introduced one of the most prominent results called Banach contraction principle and its existence in metric fixed point theory i.e., Let $J$ be a self map on a non-empty set $X$ and $d$ is a complete metric. If there exists a constant $\kappa \in[0,1)$ such that

$$
d(J \mu, J \eta) \leq \kappa d(\mu, \eta) \text { for all } \mu, \eta \in X
$$

then it possesses a unique fixed point in $X$. Due to the importance and application potential of the Banach contraction principle, this notion has been extended by several authors [2-4]. In 1994, Matthews [5] introduced the notion of partial-metric space as a part of the study of denotational semantics of dataflow networks. In 1968, the following contraction was proved by Kannan [6] i.e.,

$$
d(J \mu, J \eta) \leq \rho[d(\mu, J \mu)+d(\eta, J \eta)] \quad \text { for all } \mu, \eta \in X,
$$

where $\rho \in\left[0, \frac{1}{2}\right)$. In 2018, Karapinar [7] adopted the interpolative approach to define the generalized Kannan-type contraction on a complete metric space. We recall that a self-map $J: X \rightarrow X$ is said to be 
an interpolative Kannan type contraction for a metric space $(X, d)$, if there are constants $\rho \in[0,1)$ and $\alpha \in(0,1)$ such that

$$
d(J \mu, J \eta) \leq \rho[d(\mu, J \mu)]^{\alpha} \cdot[d(\eta, J \eta)]^{1-\alpha} \text { for all } \mu, \eta \in X \backslash \operatorname{Fix}(J)
$$

where $\operatorname{Fix}(J)=\{z \in X: J z=z\}$.

In 1972, Reich [8] generalized the concepts of Kannan and Banach, e.g., a self map $J: X \rightarrow X$ is called a Reich-contraction mapping if there are $\alpha, \beta, \gamma \in[0,1)$ and $\alpha+\beta+\gamma<1$ such that

$$
d(J \mu, J \eta) \leq \alpha d(\mu, J \mu)+\beta d(\eta, J \eta)+\gamma d(\mu, \eta) \quad \text { for all } \mu, \eta \in X .
$$

Reich-Rus-Ćirić [9-15] independently proved the next theorem and its variants i.e., a self map $J: X \rightarrow X$ is said to be a Reich-Rus-Ćirić contraction map on a complete metric space $(X, d)$ if there are $\rho \in\left[0, \frac{1}{3}\right)$ such that

$$
d(J \mu, J \eta) \leq \rho[d(\mu, \eta)+d(\mu, J \mu)+d(\eta, J \eta)]
$$

for all $\mu, \eta \in X$, then $J$ possesses a unique fixed point. Very recently, Karapinar et al. [16,17] introduced the concept of interpolative Reich-Rus-Ćirić and Hardy-Rogers type contraction and proved the following fixed point results.

Theorem 1 ([16]). In the notion of partial metric space $(X, d)$, if a mapping $J: X \rightarrow X$ is an interpolative Reich-Rus-Ćirić type contraction, i.e., there are constants $\rho \in[0,1)$ and $\alpha, \beta \in(0,1)$ such that $d(J \mu, J \eta) \leq$ $\rho[d(\mu, \eta)]^{\beta}[d(\mu, J \mu)]^{\alpha} \cdot[d(\eta, J \eta)]^{1-\alpha-\beta}$ for all $\mu, \eta \in X \backslash$ Fix $(J)$, then J owns a fixed point.

Theorem 2 ([17]). Let $(X, d)$ be a metric space. If the self-mapping $J: X \rightarrow X$ is an interpolative Hardy-Rogers type contraction i.e., there exist $\rho \in[0,1)$ and $\alpha, \beta, \gamma \in(0,1)$ with $\alpha+\beta+\gamma<1$, such that

$$
\begin{aligned}
d(J \mu, J \eta) \leq & \left.\rho[d(\mu, \eta)]^{\beta}[d(\mu, J \mu)]^{\alpha} \cdot d(\eta, J \eta)\right]^{\gamma} \\
& \cdot\left[\frac{1}{2}(d(\mu, J \eta)+d(\eta, J \mu))\right]^{1-\alpha-\beta-\gamma} \quad \text { for all } \mu, \eta \in X \backslash \operatorname{Fix}(J),
\end{aligned}
$$

then J possesses a fixed point of $X$.

In continuation, interesting work was done by many authors [18-27] which enriched this field.

The purpose of this paper was to revisit the approach of interpolative Reich-Rus-Ćirić and Hardy-Rogers type contractions to attain a common fixed point for quasi-partial b-metric spaces. Some examples are given to illustrate the new approach.

\section{Preliminaries and Definitions}

Definition 1 ([28]). A quasi-partial b-metric on a non-empty set $X$ is a function $q p_{b}: X \times X \rightarrow \mathbb{R}^{+}$such that for some real number $s \geq 1$ and all $\mu, \eta, \vartheta \in X$ :

$\left(\mathrm{QPb}_{1}\right) \quad q p_{b}(\mu, \mu)=q p_{b}(\mu, \eta)=q p_{b}(\eta, \eta)$ implies $\mu=\eta$,

$\left(\mathrm{QPb}_{2}\right) \quad q p_{b}(\mu, \mu) \leq q p_{b}(\mu, \eta)$

$\left(\mathrm{QPb}_{3}\right) \quad q p_{b}(\mu, \mu) \leq q p_{b}(\eta, \mu)$,

$\left(\mathrm{QPb}_{4}\right) \quad q p_{b}(\mu, \eta) \leq s\left[q p_{b}(\mu, \vartheta)+q p_{b}(\vartheta, \eta)\right]-q p_{b}(\vartheta, \vartheta)$.

$\left(X, q p_{b}\right)$ is called a quasi-partial b-metric space where $X$ is a non-empty set and $q p_{b}$ defines a quasi-partial $b$-metric on $X$. The number $s$ is called the coefficient of $\left(X, q p_{b}\right)$.

Let $q p_{b}$ be a quasi-partial b-metric on the set $X$. Then

$$
d_{q p_{b}}(\mu, \eta)=q p_{b}(\mu, \eta)+q p_{b}(\eta, \mu)-q p_{b}(\mu, \mu)-q p_{b}(\eta, \eta) \text { is a b-metric on } X \text {. }
$$


Let us see some new examples of quasi-partial b-metric space.

Example 1. Let $X=\left[0, \frac{\pi}{4 k}\right]$. Define the metric $q p_{b}(\mu, \eta)=\sin k|\mu-\eta|+\mu$ for any $(\mu, \eta) \in X \times X$ and $k \geq 2$.

It can be shown here that $\left(X, q p_{b}\right)$ is a quasi-partial b-metric space. Actually, if $q p_{b}(\mu, \mu)=q p_{b}(\mu, \eta)=$ $q p_{b}(\eta, \eta)$, that is, $\mu=\operatorname{sink}|\mu-\eta|+\mu=\eta$, then it is obvious that $\left(Q P b_{1}\right)$ holds for any $(\mu, \eta) \in X \times X$.

In addition, $\sin k|\mu-\eta| \geq 0$ and $\sin k|\mu-\eta| \geq|\mu-\eta|$ when $|\mu-\eta| \in\left[0, \frac{\pi}{4 k}\right]$, then $q p_{b}(\mu, \mu)=\mu \leq$ $\sin k|\mu-\eta|+\mu=q p_{b}(\mu, \eta)$

$$
\begin{aligned}
q p_{b}(\mu, \mu) & =\mu \\
& =|\mu-\eta+\eta| \\
& \leq|\mu-\eta|+|\eta| \\
& \leq \operatorname{sink}|\eta-\mu|+\eta \\
& =q p_{b}(\eta, \mu) \text { are true, hence }\left(Q P b_{2}\right) \text { and }\left(Q P b_{3}\right) \text { hold for any }(\mu, \eta) \in X \times X .
\end{aligned}
$$

Moreover, for any $\mu, \eta, \delta \in X,|\mu-\eta| \leq \frac{\pi}{4 k} \leq \frac{\pi}{2 k}$ and $[|\mu-\eta|+|\eta-\delta|] \leq \frac{\pi}{2 k}$ when $k(|\mu-\delta|+\mid \delta-$ $\mu \mid) \in\left[0, \frac{\pi}{2 k}\right]$, or $k(|\mu-\delta|+|\delta-\mu|) \leq \frac{\pi}{2}$, and since $\sin \mu$ is increasing on $\left[0, \frac{\pi}{2}\right]$, we get

$$
\begin{aligned}
q p_{b}(\mu, \eta)+q p_{b}(\delta, \delta) & =\sin k|\mu-\eta|+\mu+\delta \\
& \leq \operatorname{sink}(|\mu-\delta|+|\delta-\eta|)+\mu+\delta \\
& \leq k(|\mu-\delta|+|\delta-\eta|)+\mu+\delta \\
& \leq k \sin k|\mu-\delta|+k \sin k|\delta-\eta|+\mu+\delta \\
& =k(\sin k|\mu-\delta|+\sin k|\delta-\eta|+\mu+\delta) \\
& \leq s\left(q p_{b}(\mu, \delta)+q p_{b}(\delta, \eta)\right) \text { for all } \mu, \eta, \delta \in X
\end{aligned}
$$

and $s \geq k,\left(Q P b_{4}\right)$ holds, hence $\left(X, q p_{b}\right)$ is a quasi-partial b-metric space with $s \geq k$.

Lemma 1 ([29]). Let $\left(X, q p_{b}\right)$ be a quasi-partial b-metric space. Then the following hold:

(a) If $q p_{b}(\mu, \eta)=0$ then $\mu=\eta$.

(b) If $\mu \neq \eta$, then $q p_{b}(\mu, \eta)>0$ and $q p_{b}(\eta, \mu)>0$.

Definition 2 ([29]). Let $\left(X, q p_{b}\right)$ be a quasi-partial b-metric. Then

(i) A sequence $\left\{\mu_{n}\right\} \subset X$ converges to $\mu \in X$ if and only if

$$
q p_{b}(\mu, \mu)=\lim _{n \rightarrow \infty} q p_{b}\left(\mu, \mu_{n}\right)=\lim _{n \rightarrow \infty} q p_{b}\left(\mu_{n}, \mu\right) .
$$

(ii) A sequence $\left\{\mu_{n}\right\} \subset X$ is called a Cauchy sequence if and only if

$$
\lim _{n, m \rightarrow \infty} q p_{b}\left(\mu_{n}, \mu_{m}\right) \text { and } \lim _{m, n \rightarrow \infty} q p_{b}\left(\mu_{m}, \mu_{n}\right) \text { exist (and are finite). }
$$

(iii) The quasi partial b-metric space $\left(X, q p_{b}\right)$ is said to be complete if every Cauchy sequence $\left\{\mu_{n}\right\} \subset X$ converges with respect to $\tau_{q p_{b}}$ to a point $\mu \in X$ such that

$$
q p_{b}(\mu, \mu)=\lim _{n, m \rightarrow \infty} q p_{b}\left(\mu_{n}, \mu_{m}\right)=\lim _{m, n \rightarrow \infty} q p_{b}\left(\mu_{m}, \mu_{n}\right)
$$


(iv) A mapping $f: X \rightarrow X$ is said to be continuous at $\mu_{0} \in X$ if, for every $\varepsilon>0$, there exist

$$
\delta>0 \text { such that } f\left(B\left(\mu_{0}, \delta\right)\right) \subset B\left(f\left(\mu_{0}\right), \varepsilon\right) .
$$

Lemma 2 ([29]). Let $\left(X, q p_{b}\right)$ be a quasi-partial b-metric space and $\left(X, d_{q p_{b}}\right)$ be the corresponding b-metric space. Then $\left(X, d_{q p_{b}}\right)$ is complete if $\left(X, q p_{b}\right)$ is complete.

Definition 3 ([30]). Let $\left(X, q p_{b}\right)$ be a quasi-partial b-metric space and $J: X \rightarrow X$ be a given mapping. Then $J$ is said to be sequentially continuous at $z \in X$ if for each sequence $\left\{\mu_{n}\right\}$ in $X$ converging to $z$, we have $J \mu_{n} \rightarrow J z$, that is, $\lim _{n \rightarrow \infty} q p_{b}\left(J \mu_{n}, J z\right)=q p_{b}(J z, J z)$. Similarly, let $S: X \rightarrow X$ be a given mapping. $S$ is said to be sequentially continuous at $z \in X$ if for each sequence $\left\{\mu_{n}\right\}$ in $X$ converging to $z$, we have $\lim _{n \rightarrow \infty} q p_{b}\left(S z, S \mu_{n}\right)=q p_{b}(S z, S z)$. Then $J$ is said to be sequentially continuous on $X$ if $J$ is sequentially continuous at each $z \in X$.

\section{Main Result}

Let us discuss the main result.

Theorem 3. Let $\left(X, q p_{b}\right)$ be a complete quasi-partial b-metric space. Let $J, S: X \rightarrow X$ be self mappings. Assume that there are some $\rho \in[0,1), \alpha, \beta \in(0,1), \alpha+\beta<1$, and $s \geq 1$ such that the condition

$$
q p_{b}(J \mu, S \eta) \leq \rho\left[q p_{b}(\mu, \eta)\right]^{\beta}\left[q p_{b}(\mu, J \mu)\right]^{\alpha}\left[\frac{1}{s} q p_{b}(\eta, S \eta)\right]^{1-\alpha-\beta}
$$

is satisfied for all $\mu, \eta \in X$ such that $J \mu \neq \mu$ whenever $S \eta \neq \eta$. Then $S$ and J posses a common fixed point.

Proof. Let $\mu_{0} \in X$. Define the sequence $\left\{\mu_{n}\right\}$ by $\mu_{2 n+1}=J \mu_{2 n}, \mu_{2 n+2}=S \mu_{2 n+1}$ for all $n=0,1,2, \ldots$ If there exist $n \in 0,1,2, \ldots$ such that $\mu_{n}=\mu_{n+1}=\mu_{n+2}$ then $\mu_{n}$ is a common fixed point of $S$ and $J$. Suppose that there are no three consecutive identical terms in the sequence $\mu_{n}$ and that $\mu_{0} \neq \mu_{1}$. Now using (1), we deduce that

$$
\begin{aligned}
q p_{b}\left(\mu_{2 n+1}, \mu_{2 n+2}\right) & =q p_{b}\left(J \mu_{2 n}, S \mu_{2 n+1}\right) \\
& \left.\leq \rho\left[q p_{b}\left(\mu_{2 n}, \mu_{2 n+1}\right)\right]^{\beta} \cdot\left[q p_{b}\left(\mu_{2 n}, J \mu_{2 n}\right)\right]^{\alpha} \cdot\left[\frac{1}{s} q p_{b}\left(\mu_{2 n+1}, S \mu_{2 n+1}\right)\right]\right]^{1-\alpha-\beta} \\
& \leq \rho\left[q p_{b}\left(\mu_{2 n}, \mu_{2 n+1}\right)\right]^{\beta} \cdot\left[q p_{b}\left(\mu_{2 n}, \mu_{2 n+1}\right)\right]^{\alpha} \cdot\left[\frac{1}{s} q p_{b}\left(\mu_{2 n+1}, \mu_{2 n+2}\right)\right]^{1-\alpha-\beta} \\
& \leq \rho\left[q p_{b}\left(\mu_{2 n}, \mu_{2 n+1}\right)\right]^{\beta} \cdot\left[q p_{b}\left(\mu_{2 n}, \mu_{2 n+1}\right)\right]^{\alpha} \cdot\left[q p_{b}\left(\mu_{2 n+1}, \mu_{2 n+2}\right)\right]^{1-\alpha-\beta} \\
{\left[q p_{b}\left(\mu_{2 n+1}, \mu_{2 n+2}\right)\right]^{\alpha+\beta} } & \leq \rho\left[q p_{b}\left(\mu_{2 n}, \mu_{2 n+1}\right)\right]^{\alpha+\beta}
\end{aligned}
$$

or

$$
\left[q p_{b}\left(\mu_{2 n+1}, \mu_{2 n+2}\right) \leq \rho\left[q p_{b}\left(\mu_{2 n}, \mu_{2 n+1}\right)\right]\right.
$$

Hence,

$$
q p_{b}\left(\mu_{2 n+1}, \mu_{2 n+2}\right) \leq \rho q p_{b}\left(\mu_{2 n}, \mu_{2 n+1}\right) \leq \rho^{2} q p_{b}\left(\mu_{2 n-1}, \mu_{2 n}\right) .
$$

Thus,

$$
q p_{b}\left(\mu_{2 n+1}, \mu_{2 n+2}\right) \leq \rho^{2 n+1} q p_{b}\left(\mu_{0}, \mu_{1}\right)
$$


Similarly,

$$
\begin{aligned}
q p_{b}\left(\mu_{2 n+1}, \mu_{2 n}\right) & =q p_{b}\left(J \mu_{2 n}, S \mu_{2 n-1}\right) \\
& \leq \rho\left[q p_{b}\left(\mu_{2 n}, \mu_{2 n-1}\right)\right]^{\beta} \cdot\left[q p_{b}\left(\mu_{2 n}, J \mu_{2 n}\right)\right]^{\alpha} \cdot\left[\frac{1}{s} q p_{b}\left(\mu_{2 n-1}, S \mu_{2 n-1}\right)\right]^{1-\alpha-\beta} \\
& \leq \rho\left[q p_{b}\left(\mu_{2 n}, \mu_{2 n-1}\right)\right]^{\beta} \cdot\left[q p_{b}\left(\mu_{2 n}, \mu_{2 n+1}\right)\right]^{\alpha} \cdot\left[\frac{1}{s} q p_{b}\left(\mu_{2 n-1}, \mu_{2 n}\right)\right]^{1-\alpha-\beta} \\
{\left[q p_{b}\left(\mu_{2 n+1}, \mu_{2 n}\right)\right]^{1-\alpha} } & \leq \rho\left[q p_{b}\left(\mu_{2 n-1}, \mu_{2 n}\right)\right]^{1-\alpha} \\
{\left[q p_{b}\left(\mu_{2 n+1}, \mu_{2 n}\right)\right] } & \leq \rho\left[q p_{b}\left(\mu_{2 n-1}, \mu_{2 n}\right)\right]
\end{aligned}
$$

Hence,

$$
q p_{b}\left(\mu_{2 n+1}, \mu_{2 n}\right) \leq \rho q p_{b}\left(\mu_{2 n-1}, \mu_{2 n}\right) \leq \rho^{2} q p_{b}\left(\mu_{2 n-1}, \mu_{2 n-2}\right) .
$$

Thus,

$$
q p_{b}\left(\mu_{2 n+1}, \mu_{2 n}\right) \leq \rho^{2 n} q p_{b}\left(\mu_{0}, \mu_{1}\right) .
$$

From (2) and (3), we can deduce that

$$
q p_{b}\left(\mu_{n}, \mu_{n+1}\right) \leq \rho^{n} q p_{b}\left(\mu_{0}, \mu_{1}\right) .
$$

To prove sequence $\left\{\mu_{n}\right\}$ is Cauchy, let $n, k \in N$

$$
\begin{aligned}
q p_{b}\left(\mu_{n}, \mu_{n+k}\right) & \leq s q p_{b}\left(\mu_{n}, \mu_{n+1}\right)+s^{2} q p_{b}\left(\mu_{n+1}, \mu_{n+2}\right)+\ldots+s^{k} q p_{b}\left(\mu_{n+k-1}, \mu_{n+k}\right) \\
& \leq\left[s \rho^{n}+s^{2} \rho^{n+1}+\ldots+s^{k} \rho^{n+k-1}\right] q p_{b}\left(\mu_{0}, \mu_{1}\right) \\
& \leq s^{k} \sum_{i=n}^{n+k-1} \rho^{i} q p_{b}\left(\mu_{0}, \mu_{1}\right) \\
& \leq s^{k} \sum_{i=n}^{\infty} \rho^{i} q p_{b}\left(\mu_{0}, \mu_{1}\right)
\end{aligned}
$$

From (5),

$$
\begin{aligned}
q p_{b}\left(\mu_{n+m}, \mu_{n+m+k}\right) & \leq s^{k} \sum_{i=m}^{\infty} \rho^{i} q p_{b}\left(\mu_{n}, \mu_{n+1}\right) \\
\lim _{m \rightarrow \infty, n \rightarrow \infty} q p_{b}\left(\mu_{n+m}, \mu_{n+m+k}\right) & \leq s^{k} \lim _{m \rightarrow \infty} \sum_{i=m}^{\infty} \lim _{n \rightarrow \infty} \rho^{i} q p_{b}\left(\mu_{n}, \mu_{n+1}\right) \\
& =0
\end{aligned}
$$

Therefore,

$$
\lim _{n \rightarrow \infty} q p_{b}\left(\mu_{n}, \mu_{n+k}\right)=\lim _{m \rightarrow \infty, n \rightarrow \infty} q p_{b}\left(\mu_{n+m}, \mu_{n+m+k}\right)=0 .
$$

We conclude that $\left\{\mu_{n}\right\}$ is a Cauchy sequence. Since $\left(X, q p_{b}\right)$ is complete, there exists $z \in X$ such that $\lim _{n \rightarrow \infty} \mu_{n}=z$. Next, we shall prove that $z$ is a common fixed point of $S$ and $T$.

$$
\begin{aligned}
q p_{b}\left(J z, \mu_{2 n+2}\right) & =q p_{b}\left(J z, S \mu_{2 n+1}\right) \\
& \leq \rho\left[q p_{b}\left(z, \mu_{2 n+1}\right)\right]^{\beta} \cdot\left[q p_{b}(z, J z)\right]^{\alpha} \cdot\left[\frac{1}{s} q p_{b}\left(\mu_{2 n+1}, S \mu_{2 n+1}\right)\right]^{1-\alpha-\beta} \\
& \leq \rho\left[q p_{b}\left(z, \mu_{2 n+1}\right)\right]^{\beta} \cdot\left[q p_{b}(z, J z)\right]^{\alpha} \cdot\left[q p_{b}\left(\mu_{2 n+1}, S \mu_{2 n+1}\right)\right]^{1-\alpha-\beta}
\end{aligned}
$$


Letting $n \rightarrow \infty$, we get, $J z=z$.

Similarly,

$$
\begin{aligned}
q p_{b}\left(\mu_{2 n+1}, S z\right) & =q p_{b}\left(J \mu_{2 n}, S z\right) \\
& \leq \rho\left[q p_{b}\left(\mu_{2 n}, S z\right)\right]^{\beta} \cdot\left[q p_{b}\left(\mu_{2 n}, J \mu_{2 n}\right)\right]^{\alpha} \cdot\left[\frac{1}{s} q p_{b}(z, S z)\right]^{1-\alpha-\beta} \\
& \leq \rho\left[q p_{b}\left(\mu_{2 n}, S z\right)\right]^{\beta} \cdot\left[q p_{b}\left(\mu_{2 n}, \mu_{2 n+1}\right)\right]^{\alpha} \cdot\left[q p_{b}(z, S z)\right]^{1-\alpha-\beta}
\end{aligned}
$$

Letting $n \rightarrow \infty$, we get $S z=z$. Hence $S$ and $J$ attain a common fixed point.

The following fixed point result in the setting of complete quasi-partial b-metric space can be obtained from our main result Theorem 3.

Corollary 1. Let $\left(X, q p_{b}\right)$ be a complete quasi-partial b-metric space and $J, S: X \rightarrow X$ be self mappings such that $q p_{b}(J \mu, S \eta) \leq\left[q p_{b}(\mu, J \mu)\right]^{\alpha}\left[q p_{b}(\eta, S \eta)\right]^{1-\alpha}$ for all $\mu, \eta \in X, \rho \in[0,1), \alpha \in(0,1), J \mu \neq \mu$ whenever $S \eta \neq \eta$. Then $S$ and J own a common fixed point.

Proof. Taking $\beta=0$ in Theorem 1.

We now justify our result by illustrating it with an example below.

Example 2. Let $X=\{1,2,3,4\}$. Define complete quasi-partial b-metric as $q p_{b}(\mu, \eta)=\max \{\mu, \eta\}+|\mu-\eta|$ that is:

\begin{tabular}{ccccc}
\hline $\boldsymbol{q} p_{\boldsymbol{b}}(\boldsymbol{\mu}, \boldsymbol{\eta})$ & $\mathbf{1}$ & $\mathbf{2}$ & $\mathbf{3}$ & $\mathbf{4}$ \\
\hline $\mathbf{1}$ & 1 & 3 & 5 & 7 \\
$\mathbf{2}$ & 3 & 2 & 4 & 6 \\
$\mathbf{3}$ & 5 & 4 & 3 & 5 \\
$\mathbf{4}$ & 7 & 6 & 5 & 4 \\
\hline
\end{tabular}

We define self mappings $J$ and $S$ on $X$ as $J:\left(\begin{array}{llll}1 & 2 & 3 & 4 \\ 1 & 2 & 1 & 2\end{array}\right), S:\left(\begin{array}{llll}1 & 2 & 3 & 4 \\ 1 & 2 & 2 & 1\end{array}\right)$ as shown in Figure 1. Choose $\alpha=\frac{1}{2}, \beta=\frac{1}{3}$, and $\rho=\frac{7}{10}$.

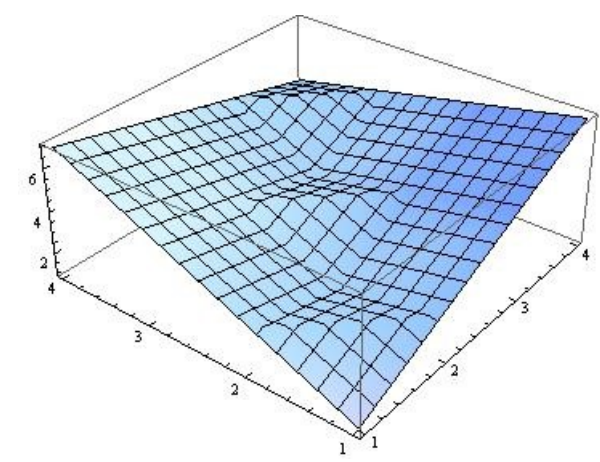

Figure 1. 1 is the common fixed point of $\mathrm{S}$ and $\mathrm{J}$.

Case 1: Let $(\mu, \eta)=(3,4)$. Without loss of generality, we have

$$
\begin{aligned}
& q p_{b}(J \mu, S \eta) \leq \rho\left[q p_{b}(\mu, \eta)\right]^{\beta}\left[q p_{b}(\mu, J \mu)\right]^{\alpha}\left[\frac{1}{s} q p_{b}(\eta, S \eta)\right]^{1-\alpha-\beta} \\
& q p_{b}(J 3, S 4)=1 \leq \rho\left[q p_{b}(3,4)\right]^{1 / 3}\left[q p_{b}(3, J 3)\right]^{1 / 2}\left[\frac{1}{s} q p_{b}(4, S 4)\right]^{1 / 6}
\end{aligned}
$$


Case 2: Let $(\mu, \eta)=(1,4)$

$$
q p(J 1, S 4)=1 \leq \rho\left[q p_{b}(1,4)\right]^{1 / 3}\left[q p_{b}(1, J 1)\right]^{1 / 2}\left[\frac{1}{s} q p_{b}(4, S 4)\right]^{1 / 6}
$$

Thus, 1 is the common fixed point of $S$ and $J$ in the setting of interpolative Reich-Rus-Ciric type contraction. Many more common fixed points can be obtained in similar manner. Hence, a fixed point exists but is not unique.

In our next theorem, we extend our obtained result for Hardy-Rogers contraction by using interpolative approach.

Theorem 4. Let $\left(X, q p_{b}\right)$ be a complete quasi-partial b-metric space. Let $J, S: X \rightarrow X$ be self mappings. Assume that there are some $\rho \in[0,1), \alpha, \beta, \gamma \in(0,1)$ with $\alpha+\beta+\gamma<1$ and $s \geq 1$ such that the condition

$$
\begin{aligned}
q p_{b}(J \mu, S \eta) \leq & \rho\left[q p_{b}(\mu, \eta)\right]^{\beta}\left[q p_{b}(\mu, J \mu)\right]^{\alpha} \cdot\left[q p_{b}(\eta, S \eta)\right]^{\gamma} \\
& \cdot\left[\frac{1}{2 s}\left(q p_{b}(\mu, S \eta)+q p_{b}(\eta, J \mu)\right)\right]^{1-\alpha-\beta-\gamma}
\end{aligned}
$$

is satisfied for all $\mu, \eta \in X$ such that $J \mu \neq \mu$ whenever $S \eta \neq \eta$. Then $S$ and J posses a common fixed point.

Proof. For any arbitrary initial point $\mu_{0} \in\left(X, q p_{b}\right)$, we construct an iterative sequence $\left\{\mu_{n}\right\}_{n \geq 1}$ by $\mu_{2 n+1}=J \mu_{2 n}, \mu_{2 n+2}=S \mu_{2 n+1}$. If there exist $n_{0} \in N$ such that $\mu_{n_{0}}=\mu_{n_{0}+1}=\mu_{n_{0}+2}$ then $\mu_{n_{0}}$ is a common fixed point of $S$ and J. Suppose that there are no three consecutive identical terms in the sequence. Substituting $\mu$ by $\mu_{2 n+1}$ and $\eta$ by $\mu_{2 n+2}$ in (7), we have

$$
\begin{aligned}
q p_{b}\left(\mu_{2 n+1}, \mu_{2 n+2}\right)= & q p_{b}\left(J \mu_{2 n}, S \mu_{2 n+1}\right) \\
\leq & \rho\left[q p_{b}\left(\mu_{2 n}, \mu_{2 n+1}\right)\right]^{\beta}\left[q p_{b}\left(\mu_{2 n}, J \mu_{2 n}\right)\right]^{\alpha} \cdot\left[q p_{b}\left(\mu_{2 n+1}, S \mu_{2 n+1}\right)\right]^{\gamma} \\
& \cdot\left[\frac{1}{2 s}\left(q p_{b}\left(\mu_{2 n}, S \mu_{2 n+1}\right)+q p_{b}\left(\mu_{2 n+1}, J \mu_{2 n}\right)\right)\right]^{1-\alpha-\beta-\gamma} \\
\leq & \rho\left[q p_{b}\left(\mu_{2 n}, \mu_{2 n+1}\right)\right]^{\beta}\left[q p_{b}\left(\mu_{2 n}, \mu_{2 n+1}\right)\right]^{\alpha} \cdot\left[q p_{b}\left(\mu_{2 n+1}, \mu_{2 n+2}\right)\right]^{\gamma} \\
& \cdot\left[\frac{1}{2 s}\left(q p_{b}\left(\mu_{2 n}, \mu_{2 n+2}\right)+q p_{b}\left(\mu_{2 n+1}, \mu_{2 n+1}\right)\right)\right]^{1-\alpha-\beta-\gamma}
\end{aligned}
$$

By $\left(\mathrm{QPb}_{1}\right)$ and $(8)$,

$$
\begin{aligned}
\leq & \rho\left[q p_{b}\left(\mu_{2 n}, \mu_{2 n+1}\right)\right]^{\beta}\left[q p_{b}\left(\mu_{2 n}, \mu_{2 n+1}\right)\right]^{\alpha} \cdot\left[q p_{b}\left(\mu_{2 n+1}, \mu_{2 n+2}\right)\right]^{\gamma} \\
& \cdot\left[\frac{1}{2 s} \cdot s\left(\left(q p_{b}\left(\mu_{2 n+2}, \mu_{2 n+1}\right)+q p_{b}\left(\mu_{2 n+1}, \mu_{2 n}\right)\right)\right)\right]^{1-\alpha-\beta-\gamma} \\
\leq & \rho\left[q p_{b}\left(\mu_{2 n}, \mu_{2 n+1}\right)\right]^{\beta}\left[q p_{b}\left(\mu_{2 n}, \mu_{2 n+1}\right)\right]^{\alpha} \cdot\left[q p_{b}\left(\mu_{2 n+1}, \mu_{2 n+2}\right)\right]^{\gamma} \\
& \cdot\left[\frac{1}{2}\left(q p_{b}\left(\mu_{2 n+2}, \mu_{2 n+1}\right)+q p_{b}\left(\mu_{2 n+1}, \mu_{2 n}\right)\right)\right]^{1-\alpha-\beta-\gamma}
\end{aligned}
$$

Suppose that

$$
\begin{aligned}
& q p_{b}\left(\mu_{2 n}, \mu_{2 n+1}\right)<q p_{b}\left(\mu_{2 n+1}, \mu_{2 n+2}\right) \\
& \frac{1}{2}\left(q p_{b}\left(\mu_{2 n+2}, \mu_{2 n+1}\right)+q p_{b}\left(\mu_{2 n+1}, \mu_{2 n}\right)\right) \leq q p_{b}\left(\mu_{2 n+1}, \mu_{2 n+2}\right) .
\end{aligned}
$$


By (8),

$$
\begin{aligned}
q p_{b}\left(\mu_{2 n+1}, \mu_{2 n+2}\right) \leq & \rho\left[q p_{b}\left(\mu_{2 n}, \mu_{2 n+1}\right)\right]^{\beta}\left[q p_{b}\left(\mu_{2 n}, \mu_{2 n+1}\right)\right]^{\alpha} \cdot\left[q p_{b}\left(\mu_{2 n+1}, \mu_{2 n+2}\right)\right]^{\gamma} \\
& \cdot\left[q p_{b}\left(x_{2 n+1}, x_{2 n+2}\right)\right]^{1-\alpha-\beta-\gamma} \\
{\left[q p_{b}\left(\mu_{2 n+1}, \mu_{2 n+2}\right)\right]^{\alpha+\beta} \leq } & \rho\left[q p_{b}\left(\mu_{2 n}, \mu_{2 n+1}\right)\right]^{\alpha+\beta}
\end{aligned}
$$

or

$$
\left[q p_{b}\left(\mu_{2 n+1}, \mu_{2 n+2}\right)\right] \leq \rho\left[q p_{b}\left(\mu_{2 n}, \mu_{2 n+1}\right)\right]
$$
we have

Therefore, we obtain $\left[q p_{b}\left(\mu_{2 n+1}, \mu_{2 n+2}\right)\right] \leq\left[q p_{b}\left(\mu_{2 n}, \mu_{2 n+1}\right)\right]$, which is a contradiction. Thus,

$$
\begin{aligned}
\frac{1}{2}\left(q p_{b}\left(\mu_{2 n+2}, \mu_{2 n+1}\right)+q p_{b}\left(\mu_{2 n+1}, \mu_{2 n}\right)\right) \leq & q p_{b}\left(\mu_{2 n+1}, \mu_{2 n}\right) \\
q p_{b}\left(\mu_{2 n+1}, \mu_{2 n+2}\right) \leq & \rho\left[q p_{b}\left(\mu_{2 n}, \mu_{2 n+1}\right)\right]^{\beta}\left[q p_{b}\left(\mu_{2 n}, \mu_{2 n+1}\right)\right]^{\alpha} \cdot\left[q p_{b}\left(\mu_{2 n+1}, \mu_{2 n+2}\right)\right]^{\gamma} \\
& \cdot\left[q p_{b}\left(\mu_{2 n+1}, \mu_{2 n}\right)\right]^{1-\alpha-\beta-\gamma} \\
{\left[q p_{b}\left(\mu_{2 n+1}, \mu_{2 n+2}\right)\right]^{1-\gamma} \leq } & \rho\left[q p_{b}\left(\mu_{2 n}, \mu_{2 n+1}\right)\right]^{1-\gamma}
\end{aligned}
$$

We deduce that

$$
\begin{aligned}
{\left[q p_{b}\left(\mu_{2 n+1}, \mu_{2 n+2}\right)\right.} & \leq \rho\left[q p_{b}\left(\mu_{2 n}, \mu_{2 n+1}\right)\right] \\
q p_{b}\left(\mu_{2 n+1}, \mu_{2 n+2}\right) & \leq \rho q p_{b}\left(\mu_{2 n+1}, \mu_{2 n+2}\right) \leq \rho^{2}\left[q p_{b}\left(\mu_{2 n+1}, \mu_{2 n+2}\right)\right. \\
& \leq \rho^{3}\left[q p_{b}\left(\mu_{2 n+1}, \mu_{2 n+2}\right) \rho^{2 n+1} q p_{b}\left(\mu_{0}, \mu_{1}\right)\right. \\
q p_{b}\left(\mu_{2 n+1}, \mu_{2 n+2}\right) & \leq \rho^{2 n+1} q p_{b}\left(\mu_{0}, \mu_{1}\right) .
\end{aligned}
$$

Similarly

$$
\begin{aligned}
& q p_{b}\left(\mu_{2 n+1}, \mu_{2 n}\right)= q p_{b}\left(J \mu_{2 n}, S \mu_{2 n-1}\right) \\
& \leq \rho\left[q p_{b}\left(\mu_{2 n}, \mu_{2 n-1}\right)\right]^{\beta}\left[q p_{b}\left(\mu_{2 n}, J x_{2 n}\right)\right]^{\alpha} \cdot\left[q p_{b}\left(\mu_{2 n-1}, S \mu_{2 n-1}\right)\right]^{\gamma} \\
& \cdot\left[\frac{1}{2 s}\left(q p_{b}\left(\mu_{2 n}, S \mu_{2 n-1}\right)+q p_{b}\left(\mu_{2 n-1}, J \mu_{2 n}\right)\right)\right]^{1-\alpha-\beta-\gamma} \\
& \leq \rho\left[q p_{b}\left(\mu_{2 n}, \mu_{2 n-1}\right)\right]^{\beta}\left[q p_{b}\left(\mu_{2 n}, \mu_{2 n+1}\right)\right]^{\alpha} \cdot\left[q p_{b}\left(\mu_{2 n-1}, \mu_{2 n}\right)\right]^{\gamma} \\
& \cdot\left[\frac{1}{2 s}\left(q p_{b}\left(\mu_{2 n}, \mu_{2 n}\right)+q p_{b}\left(\mu_{2 n-1}, \mu_{2 n+1}\right)\right)\right]^{1-\alpha-\beta-\gamma} \\
& \leq \rho\left[q p_{b}\left(\mu_{2 n}, \mu_{2 n-1}\right)\right]^{\beta}\left[q p_{b}\left(\mu_{2 n}, \mu_{2 n+1}\right)\right]^{\alpha} \cdot\left[q p_{b}\left(\mu_{2 n-1}, \mu_{2 n}\right)\right]^{\gamma} \\
& \cdot\left[\frac{1}{2 s} \cdot s\left(\left(q p_{b}\left(\mu_{2 n}, \mu_{2 n+1}\right)+q p_{b}\left(\mu_{2 n-1}, \mu_{2 n}\right)\right)\right)\right]^{1-\alpha-\beta-\gamma} \\
& \leq \rho\left[q p_{b}\left(\mu_{2 n}, \mu_{2 n-1}\right)\right]^{\beta}\left[q p_{b}\left(\mu_{2 n}, \mu_{2 n+1}\right)\right]^{\alpha} \cdot\left[q p_{b}\left(\mu_{2 n-1}, \mu_{2 n}\right)\right]^{\gamma} \\
& \cdot\left[\frac{1}{2}\left(q p_{b}\left(\mu_{2 n}, \mu_{2 n+1}\right)+q p_{b}\left(\mu_{2 n-1}, \mu_{2 n}\right)\right)\right]^{1-\alpha-\beta-\gamma} \\
& \frac{1}{2}\left(q p_{b}\left(\mu_{2 n+2}, \mu_{2 n+1}\right)+\right.\left.q p_{b}\left(\mu_{2 n+1}, \mu_{2 n}\right)\right) \leq q p_{b}\left(\mu_{2 n-1}, \mu_{2 n}\right) \\
& q p_{b}\left(\mu_{2 n+1}, \mu_{2 n}\right) \leq \rho\left[q p_{b}\left(\mu_{2 n}, \mu_{2 n-1}\right)\right]^{\beta}\left[q p_{b}\left(\mu_{2 n}, \mu_{2 n+1}\right)\right]^{\alpha} \\
& \cdot {\left[q p_{b}\left(\mu_{2 n-1}, \mu_{2 n}\right)\right]^{\gamma} \cdot\left[q p_{b}\left(\mu_{2 n-1}, \mu_{2 n}\right)\right]^{1-\alpha-\beta-\gamma} } \\
& {\left[q p_{b}\left(\mu_{2 n+1}, \mu_{2 n}\right)\right]^{1-\gamma} \leq \rho\left[q p_{b}\left(\mu_{2 n-1}, \mu_{2 n}\right)\right]^{1-\gamma} }
\end{aligned}
$$


We deduce that

$$
\begin{aligned}
{\left[q p_{b}\left(\mu_{2 n+1}, \mu_{2 n}\right)\right.} & \leq \rho\left[q p_{b}\left(\mu_{2 n-1}, \mu_{2 n}\right)\right] \\
q p_{b}\left(\mu_{2 n+1}, \mu_{2 n}\right) & \leq \rho q p_{b}\left(\mu_{2 n-1}, \mu_{2 n}\right) \leq \rho^{2}\left[q p_{b}\left(\mu_{2 n-2}, \mu_{2 n-1}\right) \leq \cdots \leq \rho^{2 n} q p_{b}\left(\mu_{0}, \mu_{1}\right)\right. \\
q p_{b}\left(\mu_{2 n+1}, \mu_{2 n}\right) & \leq \rho^{2 n} q p_{b}\left(\mu_{0}, \mu_{1}\right)
\end{aligned}
$$

By (6), sequence $\left\{\mu_{n}\right\}$ is Cauchy. By completeness property, there exists $z \in X$ such that

$$
\lim _{n \rightarrow \infty} \mu_{n}=z
$$

Again, we shall show that $S$ and $T$ attain $\mathrm{z}$ as a common fixed point of $X$.

$$
\begin{aligned}
q p_{b}\left(J z, \mu_{2 n+2}\right)= & q p_{b}\left(J z, S \mu_{2 n+1}\right) \\
\leq & \rho\left[q p_{b}\left(z, \mu_{2 n+1}\right)\right]^{\beta}\left[q p_{b}(z, J z)\right]^{\alpha} \cdot\left[q p_{b}\left(\mu_{2 n+1}, S \mu_{2 n+1}\right)\right]^{\gamma} \\
& \cdot\left[\frac{1}{2 s}\left(q p_{b}\left(z, S \mu_{2 n+1}\right)+q p_{b}\left(\mu_{2 n+1}, J z\right)\right)\right]^{1-\alpha-\beta-\gamma} \\
\leq & \rho\left[q p_{b}\left(z, \mu_{2 n+1}\right)\right]^{\beta}\left[q p_{b}(z, T z)\right]^{\alpha} \cdot\left[q p_{b}\left(\mu_{2 n+1}, \mu_{2 n+2}\right)\right]^{\gamma} \\
& \cdot\left[\frac{1}{2 s}\left(q p_{b}\left(z, \mu_{2 n+2}\right)+q p_{b}\left(\mu_{2 n+1}, J z\right)\right)\right]^{1-\alpha-\beta-\gamma}
\end{aligned}
$$

Letting $n \rightarrow \infty$, we get, $J z=z$.

Similarly

$$
\begin{aligned}
q p_{b}\left(\mu_{2 n+1}, S z\right)= & q p_{b}\left(J \mu_{2 n}, S z\right) \\
\leq & \rho\left[q p_{b}\left(\mu_{2 n}, z\right)\right]^{\beta}\left[q p_{b}\left(x_{2 n}, J \mu_{2 n}\right)\right]^{\alpha} \cdot\left[q p_{b}(z, S z)\right]^{\gamma} \\
& \cdot\left[\frac{1}{2 s}\left(q p_{b}\left(\mu_{2 n}, S z\right)+q p_{b}\left(z, J \mu_{2 n}\right)\right)\right]^{1-\alpha-\beta-\gamma}
\end{aligned}
$$

Letting $n \rightarrow \infty$, we get $S z=z$. Hence self mappings $S$ and $J$ posses common fixed points in complete metric space.

The above result (Theorem 3) motivates us to generalize the interpolative Reich-Rus-Ćirić contraction for a family of maps. More precisely:

Problem 1. Let $\left(X, q p_{b}\right)$ be a complete quasi-partial b-metric space. Consider a family of self maps $S_{n}: X \rightarrow X$, $n \geq 1$, and $s \geq 1$ such that

$$
q p_{b}\left(S_{i} \mu, S_{j} \eta\right) \leq \rho_{i, j}\left[q p_{b}(\mu, \eta)\right]^{\beta_{j}} \cdot\left[q p_{b}(\mu, J \mu)\right]^{\alpha_{i}} \cdot\left[\frac{1}{s} q p_{b}\left(\eta, S_{\eta}\right)\right]^{1-\alpha_{i}-\beta_{j}}
$$

What are the conditions on $\rho_{i, j}, \alpha_{i}, \beta_{j}$ for $S_{n}$ to have a common fixed point?

\section{Conclusions}

The significant contribution of the paper proves the existence of common fixed points for interpolative Reich-Rus-Ćirić and Hardy-Rogers contraction mappings on quasi-partial b-metric space. Many real world problems and experimental signals lack a sensation of smoothness in their traces. Therefore, to model these signals, interpolants are required that are non-differentiable in a dense set of points in the domain. Fractal interpolation, which is based on the theory of iterated function system, is used to solve such problems. The uniqueness property of fixed points for these mappings 
and their application in the study of nonlinear integral equations will be an interesting concept for subsequent work.

Author Contributions: Conceptualization, V.N.M., L.M.S.R., P.G. and S.V.; investigation, V.N.M., L.M.S.R., P.G. and S.V.; methodology, V.N.M., L.M.S.R., P.G. and S.V.; writing-review editing, V.N.M., L.M.S.R., P.G. and S.V. All authors contributed equally and significantly in writing this article. All authors have read and agreed to the published version of the manuscript.

Funding: This research received no external funding.

Acknowledgments: The authors would like to thank the anonymous referees for their careful reading, valuable comments and suggestion that helps to improve the manuscript.

Conflicts of Interest: The authors declare that they have no competing interests.

\section{References}

1. Banach, S. Sur les opérationsdans les ensembles abstraits et leur application aux équationsintégrales. Fund Math. 1922, 3, 133-181. [CrossRef]

2. Bakhtin, I.A. The contraction principle in quasi-metric spaces. Int. Funct. Anal. 1989, 30, $26-37$.

3. Czerwik, S. Contraction mappings in b-metric spaces. Acta Math. Inform. Univ. Ostrav. 1993, 1, 5-11.

4. Shukla, S. Partial b-metric spaces and fixed point theorems. Mediterr. J. Math. 2014, 11, 703-711. [CrossRef]

5. Matthews, S.G. Partial metric topology. Ann. N. Y. Acad. Sci. 1994, 728, 183-197. [CrossRef]

6. Kannan, R. Some results on fixed points. Bull. Calcutta Math. Soc. 1968, 60, 71-76.

7. Karapinar, E. Revisiting the Kannan type contractions via interpolation. Adv. Theory Nonlinear Anal. Appl. 2018, 2, 85-87. [CrossRef]

8. Reich, S. Fixed point of contractive functions. Bollettino dell'Unione Matematica Italiana 1972, 4, $26-42$.

9. Ćirić, L.B. On contraction type mappings. Math. Balk. 1971, 1, 52-57.

10. Ćirić, L.B. Generalized contractions and fixed-point theorems. Publ. Inst. Math. (Beograd) 1971, 12, 19-26.

11. Reich, S. Some remarks concerning contraction mappings. Can. Math. Bull. 1971, 14, 121-124. [CrossRef]

12. Reich, S. Kannan's fixed point theorem. Boll. dell'Unione Mat. Ital. 1971, 4, 1-11.

13. Rus, I.A. Generalized Contractions and Applications; Cluj University Press: Clui-Napoca, Romania, 2001.

14. Rus, I.A. Principles and Applications of the Fixed Point Theory; Editura Dacia: Clui-Napoca, Romania, 1979. (In Romanian)

15. Hardy, G.E.; Rogers, T.D. A generalization of a fixed point theorem of Reich. Can. Math. Bull. 1973, 16, 201-206. [CrossRef]

16. Karapinar, E.; Agarwal, R.P.; Aydi, H. Interpolative Reich-Rus-Ćirić type contractions on partial-metric spaces. Mathematics 2018, 6, 256. [CrossRef]

17. Karapinar, E.; Alqahtani, O.; Aydi, H. On interpolative Hardy-Rogers type contractions. Symmetry 2018, 11, 8. [CrossRef]

18. Aydi, H.; Karapinar, E.; Hierro, A.F.R. $\omega$-Interpolative Reich-Rus-Ćirić type contractions. Mathematics 2019, 7, 57. [CrossRef]

19. Debnath, P.; de La Sen, M. Set-valued interpolative Hardy-Rogers and set-valued Reich-Rus-Ćirić-type contractions in b-metric spaces. Mathematics 2019, 7, 849. [CrossRef]

20. Karapinar, E.; Agarwal, R.P. Interpolative Reich-Rus-Ćirić type contractions via simulation functions. Mathematics 2018, 6, 256. [CrossRef]

21. Alqahtani, B.; Fulga, A.; Karapinar, E. Fixed Point Results on $\triangle$-Symmetric Quasi-Metric Space via Simulation Function with an Application to Ulam Stability. Mathematics 2018, 6, 208. [CrossRef]

22. Aydi, H.; Chen, C.-M.; Karapınar, E. Interpolative Reich-Rus-Ćirić Type Contractions via the Branciari Distance. Mathematics 2019, 7, 84. [CrossRef]

23. Aydi, H.; Karapınar, E. A Meir Keeler common type fixed point theorem on partial metric spaces. Fixed Point Theory Appl. 2012, 2012, 26. [CrossRef]

24. Ćirić, L.J.; Samet, B.; Aydi, H.; Vetro, C. Common fixed points of generalized contractions on partial-metric spaces and an application. Appl. Math. Comput. 2011, 218, 2398-2406. [CrossRef]

25. Karapınar, E.; Chi, K.P.; Thanh, T.D. A generalization of Ciric quasi-contractions. Abstr. Appl. Anal. $2012,2012$. [CrossRef] 
26. Mlaiki, N.; Abodayeh, K.; Aydi, H.; Abdeljawad, T.; Abuloha, M. Rectangular metric-like type spaces and related fixed points. J. Math. 2018, 2018. [CrossRef]

27. Krein, S.G.; Petunin, J.I.; Semenov, E.M. Interpolation of Linear Operators; American Mathematical Society: Providence, RI, USA, 1978.

28. Gupta, A.; Gautam, P. Topological structure of quasi-partial b-metric spaces. Int. J. Pure Math. Sci. 2016, 17, 8-18. [CrossRef]

29. Gupta, A.; Gautam, P. Quasi partial b-metric spaces and some related fixed point theorems. Fixed Point Theory Appl. 2015, 2015, 18. [CrossRef]

30. Gautam, P.; Mishra,V.N.; Negi, K. Common fixed point theorems for cyclic Reich-Rus-Ćirić contraction mappings in quasi-partial b-metric space. Ann. Fuzzy Math. Inf. 2020, accepted.

(C) 2020 by the authors. Licensee MDPI, Basel, Switzerland. This article is an open access article distributed under the terms and conditions of the Creative Commons Attribution (CC BY) license (http:// creativecommons.org/licenses/by/4.0/). 